\title{
Updating Displays After Imagined Object and Viewer Rotations
}

\author{
Maryjane Wraga, Sarah H. Creem, and Dennis R. Proffitt \\ University of Virginia
}

\begin{abstract}
Six experiments compared spatial updating of an array after imagined rotations of the array versus viewer. Participants responded faster and made fewer errors in viewer tasks than in array tasks while positioned outside (Experiment 1) or inside (Experiment 2) the array. An apparent array advantage for updating objects rather than locations was attributable to participants imagining translations of single objects rather than rotations of the array (Experiment 3). Superior viewer performance persisted when the array was reduced to 1 object (Experiment 4); however, an object with a familiar configuration improved object performance somewhat (Experiment 5). Object performance reached near-viewer levels when rotations included haptic information for the tuming object. The researchers discuss these findings in terms of the relative differences in which the human cognitive system transforms the spatial reference frames corresponding to each imagined rotation.
\end{abstract}

Suppose you are playing a board game with a group of friends, and you want to know what the board looks like from one of their perspectives, without moving to it. There are two obvious ways to proceed. You could imagine rotating the board until the side corresponding to the new perspective is coincident with your current viewpoint (object rotation). Alternatively, you could imagine moving yourself to the vantage point of the new perspective (viewer rotation). Both operations have been implicated in human beings' ability to update objects and scenes across views (e.g., Huttenlocher \& Presson, 1979; Presson, 1982; Tarr, 1995; Tarr \& Pinker, 1989). Each requires the implementation of different spatial frames of reference.

In general, frames of reference provide a locational structure within which the position of objects and events is specified. Rotation of the viewer involves movement of the egocentric frame of reference, which specifies the up/down, front/back, and left/right axes of the observer's body (e.g., Howard, 1982). Rotation of an object involves movement of its object-relative frame of reference, which specifies the location of an object's parts with respect to each other, or of one object with respect to others (e.g., Easton \& Sholl, 1995). For an object with predefined sides, such as a house, the object-relative frame delimits its intrinsic up/down, front/back, and left/right axes. For objects that do not have predetermined sides, such as a wheel or an array, assignment

Maryjane Wraga, Sarah H. Creem, and Dennis R. Proffitt, Department of Psychology, University of Virginia.

This research was supported by National Institute of Mental Health Grants MH11462 and MH52640.

We wish to thank Mackenzie Carpenter, Valerie Hung, Melissa Russano, Jeanine Stefanucci, and Wendy Zosh for assistance in data collection.

Correspondence concerning this article should be addressed to Maryjane Wraga, who is now at Harvard University, Department of Psychology, 33 Kirkland Street, Room 840, Cambridge, Massachusetts 02138. Electronic mail may be sent to mjwraga@wjh. harvard.edu. of the object-relative reference frame is extrapolated from other reference frames. For example, the top of a wheel can be determined from the gravitational vertical; the front of a wheel can be defined with respect to viewer position.

Let us return to the board game example. Updating the board's spatial configuration across imagined view changes requires alignment of the physical reference frame with the projected reference frame (i.e., the reference frame corresponding to the new view). There is evidence that the alignment process occurs via mental rotation, for both object (e.g., Shepard \& Metzler, 1971) and viewer movement (e.g., Easton \& Sholl, 1995; Rieser, 1989). Whether the transformation processes of each respective rotation are treated differently by the human cognitive system is an unresolved theoretical issue. Until recently most research has focused predominantly on updating during imagined object rotations. For example, it has been well established that observers mentally rotate one object into congruence with another in deciding whether two objects are similar in shape (e.g., Cooper, 1975; Shepard \& Metzler, 1971).

However, there is some evidence that spatial updating may be superior during self-movement than during object movement (Simons \& Wang, 1998; Wang \& Simons, 1999; Wraga, Creem, \& Proffitt, 1999b). For example, Simons and Wang (1998) found that participants' recognition of a configuration of objects was affected differently depending on whether the display or the observer was physically moved. Participants' detection of layout changes was unaffected by changes in viewpoint produced by observer movement; however, performance deteriorated when the changes were caused by rotations of the display itself. This finding held even when all environmental information (except the individual objects of the display) was eliminated to reduce possible reliance on landmarks. It also held when participants were able to actively control the rotation of the display (Wang \& Simons, 1999). Evidently, self-movement provided more effective information for updating the display than did movement of the display itself.

A growing body of empirical evidence suggests that 
objects and scenes may also be initially encoded with respect to the egocentric reference system (Diwadkar \& McNamara, 1997; Easton \& Sholl, 1995; Franklin \& Tversky, 1990; Tarr, 1995; Tarr \& Pinker, 1989). For example, Tarr (1995) found that the time it took participants to recognize a previously seen Shepard-Metzler-like object varied as a function of the angular disparity between the initial and tested views. This finding held even for subsequent presentations of the object at intermediate displacements: Response time was again related to the angular disparity from the nearest familiar perspective. Evidence for view-specific encoding has also been extended to arrays of objects (Diwadkar \& McNamara, 1997) and to representations of scenes constructed from verbal descriptions (Franklin \& Tversky, 1990).

Several researchers have directly compared updating performance for imagined viewer and object rotations (e.g., Amorim \& Stucchi, 1997; Huttenlocher \& Presson, 1979; Presson, 1982; Rock, Wheeler, \& Tudor, 1989); however, the results have been inconsistent. For example, Presson (1982) found that performance of the two transformations depended on the type of information elicited in the updating tasks. Participants typically stood before an array of objects and imagined either themselves rotating around the array or the array itself rotating. In one experiment, participants were tested with position questions. Following the imagined rotation, they stated the location of a named object (e.g., "If you/the table were rotated $90^{\circ}$, where would the drum be?"). For this type of question, the viewer task yielded slower response times and more errors than the array task, as well as more egocentric errors. A similar trend was found when the array had to be reconstructed with blocks after the respective imagined movements (appearance questions). However, a third experiment yielded the opposite result. Participants were tested with item questions, in which they named the object that would be present at a prescribed location in the array after imagined movement (e.g., "If you/the table were rotated $90^{\circ}$, what object would be on the right?"). For this type of question, the viewer task elicited fewer errors than the array task, and fewer errors were egocentric. Similar patterns of results have been found with children (Huttenlocher \& Presson, 1979; Newcombe \& Huttenlocher, 1992).

Presson and colleagues have interpreted these findings as evidence that adults and children do not encode objects with respect to the self or to other objects but rather to landmarks in the environment. By this account, the viewer positionquestion task causes a conflict between the primary (i.e., physical) array-environment relationship and the secondary (i.e., depicted) array-environment relationship. Such a conflict leads to increased response latencies and egocentric errors. The conflict is eliminated in the viewer item-question task because the imagined observer is treated as an object that is encoded and updated with respect to the environment (Huttenlocher \& Presson, 1979; Presson, 1980, 1982, 1987).

A recent adult study found a viewer advantage in a task that appears to be independent of environmental encoding (Amorim \& Stucchi, 1997). Participants imagined that a computer-displayed $F$ was standing in the center of an imaginary clock and then performed one of two tasks. In the viewer task, they imagined themselves rotating to a specified location around the clock and judged where the letter would face relative to their new position. In the object task, they imagined the letter rotating to a specified location and judged the position of themselves relative to the letter's new location. Decreased reaction times and response errors in the viewer task indicated that participants found it easier to perform than the object task. It is difficult to interpret this finding in the context of reliance on landmarks because both the array and object were imagined.

\section{Overview of the Studies}

To examine these issues further, we conducted a series of experiments that compared performance on imagined object and viewer rotations using variations of Presson and colleagues' tasks (Huttenlocher \& Presson, 1979; Presson, 1982). Observers were presented with multiple- and singleobject configurations and asked to update them after imagined rotations of the self and of the configuration. Our specific aim was to clarify the conditions under which one type of imagined movement facilitates updating over the other. Our approach was twofold: We first sought to replicate the findings of Presson and colleagues and then to extend these findings to several novel situations.

In designing the experiments, we considered the two transformations in terms of the different spatial reference frames underlying each. Imagined self-rotations require transformation of the egocentric frame. We speculated that the human cognitive system might represent this transformation process cohesively because of the biological integrity of the physical body itself. When one moves, one's entire body moves; individual axes of the egocentric frame cannot be separated. On the other hand, imagined object rotations require transformation of the object-relative frame. We speculated that such a transformation might be more difficult to represent because of the relative absence of internal cohesion, especially for objects without predetermined sides. According to this hypothesis, imagined object rotations would be made easier with progressive internal cohesion of the object-relative reference frame. That is, as the relationships among an object's up/down, front/back, and right/left axes become more apparent, performance of imagined object rotations should improve.

We conducted six studies to test this hypothesis. The first two were variations on Presson's (1982) item task. Participants were presented with a life-size array of four objects and were required to name objects in given positions after either imagining the array rotating around its central axis (array task) or imagining themselves rotating around the array (viewer task). In Experiment 1, participants were positioned outside the array; in Experiment 2, they were positioned inside the array. Like Presson, we found an advantage for imagined egocentric rotations. In Experiment 3 , we conducted a variation of Presson's position task. Imagined viewer and array rotations were made in the context of locating a specific object in the array. An additional catch-trial task presented randomly throughout the trials was designed to test whether participants in the 
array task were updating the entire array or merely the specified object. We found that performance in the position task was more or less equivalent actoss array and viewer rotation conditions. However, the results of the subsidiary task suggested that participants did not rotate the entire array in the array condition.

Experiments 4-6 examined whether the relative difficulties in the array task would dissipate as a function of increasing internal cohesion of the relationship among object parts. In Experiment 4, the four elements of the array were collapsed into one object, a block. Despite this manipulation, an advantage for viewer rotations was still found. In Experiment 5, the block was replaced with a more familiar object: a car. A viewer advantage was still found; however, performance in imagining rotation of the car was significantly improved over that of the block. In the final experiment, object-rotation performance improved to nearviewer levels with the introduction of haptically felt physical movement into the rotation task.

\section{General Method}

Six experiments were conducted. This section describes aspects of the method common to all. Details specific to each experiment are included within their individual sections.

\section{Array Materials (Experiments 1, 2, and 3)}

The array consisted of four wooden stands, $92 \mathrm{~cm}$ in height. Each stand was composed of two wooden disks $18 \mathrm{~cm}$ in diameter attached together with an $88 \mathrm{~cm}$-long, $2 \mathrm{~cm}$-diameter wooden dowel. The stands were positioned to form a diamond-shaped array, each side of which measured $75 \mathrm{~cm}$. Each stand held one plastic toy: a $15 \times 18 \mathrm{~cm}$ blue hammer, a $12 \times 4 \mathrm{~cm}$ orange phone, a $15 \times$ $7 \mathrm{~cm}$ white racecar, or a $41 \times 1 \mathrm{~cm}$ yellow snake.

\section{Object Materials (Experiments 4, 5, and 6)}

Two different objects were used. For Experiments 4 and 6, a $13 \times 8 \times 3 \mathrm{~cm}$ wooden block was used. A $8 \times 2.5 \mathrm{~cm}$ area of each of the block's edges was painted a different color: red, yellow, green, and blue. In Experiment 5, a $15 \times 7 \times 3 \mathrm{~cm}$ blue plastic race car was used. Its hood was marked with a black $H$, its trunk with a $T$, its left side with a $D$ (driver), and its right side with a $P$ (passenger). Reaction times were recorded using a Timex chronograph stopwatch.

\section{Procedure}

Each participant performed in two conditions, each with a different array/object configuration. Participants stood with heels $75 \mathrm{~cm}$ from the closest stand. They first learned the items of a given configuration in terms of top, bottom, left, and right positions in the array (see Figure 1). They were given as much time as necessary to memorize the configuration with eyes open and were then tested for the item corresponding to each position with eyes closed. Criteria for learning were met if participants could identify the position of each item correctly within one second. The experimenter then explained the task. For the viewer task, participants imagined themselves rotating (in either a clockwise or counterclockwise direction) around the outside of the array/object while maintaining the same relationship to the array/object, that is, facing inward; for top

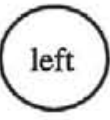

right

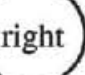

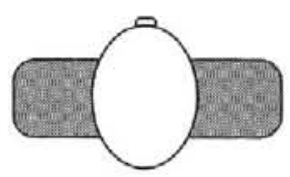

Figure 1. Overhead view of the positions of the array. Each circle represents a stand on which is placed one of four objects.

the array/object task, they imagined rotating the array/object itself in the same direction. The degrees of rotation were $0,90,180$, and 270. Before testing began, participants were blindfolded. For each trial, they received the degree of rotation followed by a position in the array/object, for example, "180, what is on the right?" They responded by naming one of the objects. They were instructed to answer as quickly and accurately as possible. Reaction times (RTs) and responses were reconded. RT was measured from the end of the experimenter's question to the beginning of the participant's response. At the end of the first condition, the array/object was arranged into a new configuration and the procedure was repeated.

\section{Design}

The order of tasks (viewer, array) and directions of rotation (clockwise, counterclockwise) were counterbalanced across participants. Participants performed both tasks using only one or the other of the rotation directions. Each of the positions (top, bottom, left, right) was matched with each of the rotations $(0,90,180,270)$, for a total of 16 trials per task. Trials were presented randomly.

\section{Analyses}

Response latency and number of errors were collected for each experiment. A log transformation was applied to the latency data in each experiment to provide a more symmetrical distribution. A square root transformation was applied to the accuracy data to correct for positive skew in the distribution. A 2 (sex) $\times 2$ (rotation direction) $\times 2$ (task onder) $\times 2$ (task) $\times 4$ (degree) mixed-design analysis of variance (ANOVA) was performed on the logtransformed latency data and on the square-root-transformed accuracy data for each experiment. To assess whether there was a differential effect of position (i.e., top, bottom, left, right) a second 2 (sex) $\times 2$ (rotation direction) $\times 2$ (task order) $\times 2$ (task) $\times 2$ 
(position: top/bottom vs. right/left) mixed-design ANOVA was performed on the latency data and on the error data.

\section{Experiment 1}

The first study was a variation of Presson's (1982) item-question experiment. He found that imagined rotations of a miniature array of objects (array task) were slower and more difficult than imagined rotations of the viewer (viewer task) around the array when the task involved item questions. These required the participant to identify an object at a specific location in the array, after performing a given imagined rotation (e.g., "rotate yourself/the table $180^{\circ}$; what's on the right?"). Our experiment tested the same question format for both array and viewer tasks. However, we placed participants before a large-size array to eliminate scale differences between the normal size of the observer and the miniature proportions of Presson's array. The dimensions of the new setup afforded actual traverseability around the array. We predicted that judgments would be faster and more accurate in the viewer task than in the array task.

\section{Method}

\section{Participants}

Twenty-four University of Virginia undergraduates (14 women, 10 men) participated in the experiment as part of a research credit requirement. Three additional participants were excluded from the experiment, 1 because of experimenter error and 2 for having more than a $50 \%$ error rate in the array task. All participants were tested individually; none knew of the hypothesis being tested.

\section{Materials}

The four-object array was used.

\section{Procedure}

See Procedure, General Method.

\section{Design}

See Design, General Method.

\section{Results}

The principal finding was that participants were faster to respond and made fewer errors in the viewer task compared with the array task.

\section{Latency}

Figure 2 shows mean RTs and standard errors for correct responses in each task as a function of degree of rotation. ${ }^{1}$ Overall $\mathrm{RT}$ in the viewer condition $(M=1.64 \mathrm{~s})$ was faster than in the array condition $(M=2.97 \mathrm{~s}){ }^{2}$ The ANOVA performed on the mean scores yielded main effects of task, $F(1,16)=43.92, p<.0001$, and degree, $F(3,48)=43.57$, $p<.0001$, and a significant Task $\times$ Degree interaction, $F(3$,

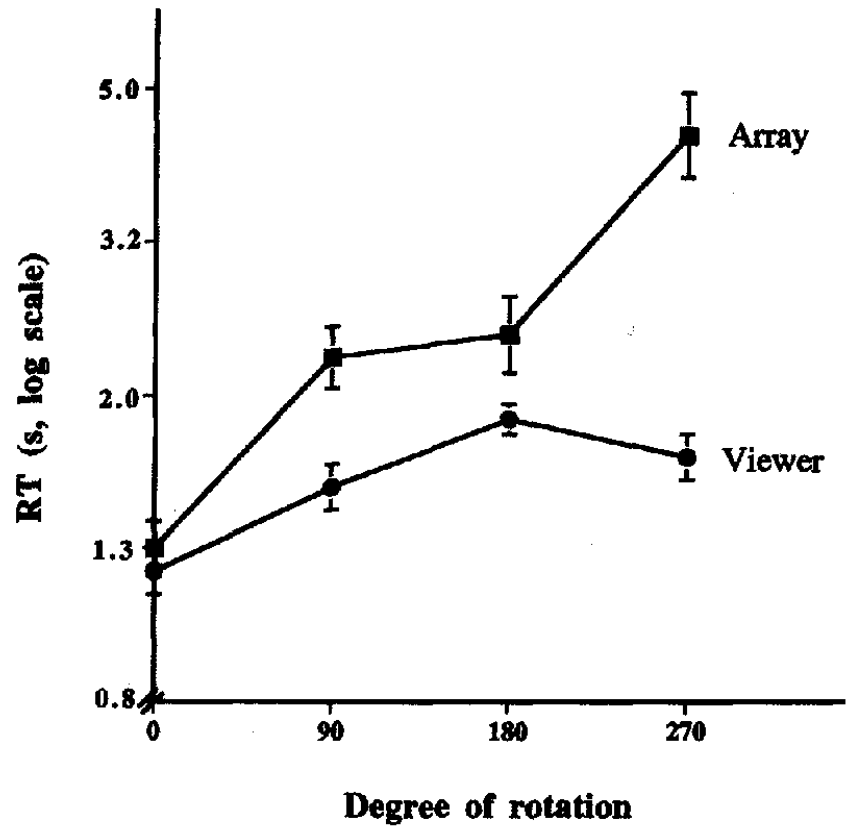

Figure 2. Mean reaction times (RT) and standard errors, Experiment 1 . Values are in seconds, depicted in log space.

$48)=15.39, p<.0001$. Contrast comparisons revealed that this interaction was a result of different RT functions across degrees for each task (see Figure 2). Notably, in the array task, RT increased from $0^{\circ}$ to $90^{\circ}$ and $180^{\circ}$ to $270^{\circ}$, whereas in the viewer task, RT increased to $180^{\circ}$ but then decreased at $270^{\circ}$.

\section{Accuracy}

In general, participants were more accurate in the viewer condition $(M=0.23$ errors) than in the array condition $(M=0.75$ errors). Figure 3 shows mean errors and standard errors as a function of task and degree of rotation. The ANOVA performed on number of errors yielded main effects of task, $F(1,16)=17.12, p<.001$, and degree, $F(3,48)=$ $8.03, p<.0001$, and a significant Task $\times$ Degree interaction, $F(3,48)=10.62, p<.0001$. An assessment of the Task $X$ Degree interaction revealed an increase in array errors for $90^{\circ}$ and $270^{\circ}$ but no effect of degree in viewer.

Egocentric errors were assessed in two ways. Because errors in each task were not necessarily produced by the same participants, the corresponding data were analyzed as separate groups. We first calculated the average egocentric errors of participants who made errors, for each task. No difference was found between the number of egocentric

\footnotetext{
${ }^{1}$ Mean RTs that were greater than three standard deviations above the overall mean of a given array or viewer degree condition were replaced with the group mean for that condition (e.g., array 270). In Experiment 1, two array RTs were replaced.

${ }^{2}$ Although mean $\log$ RT was analyzed, mean scores are reported in seconds for clarity.
} 


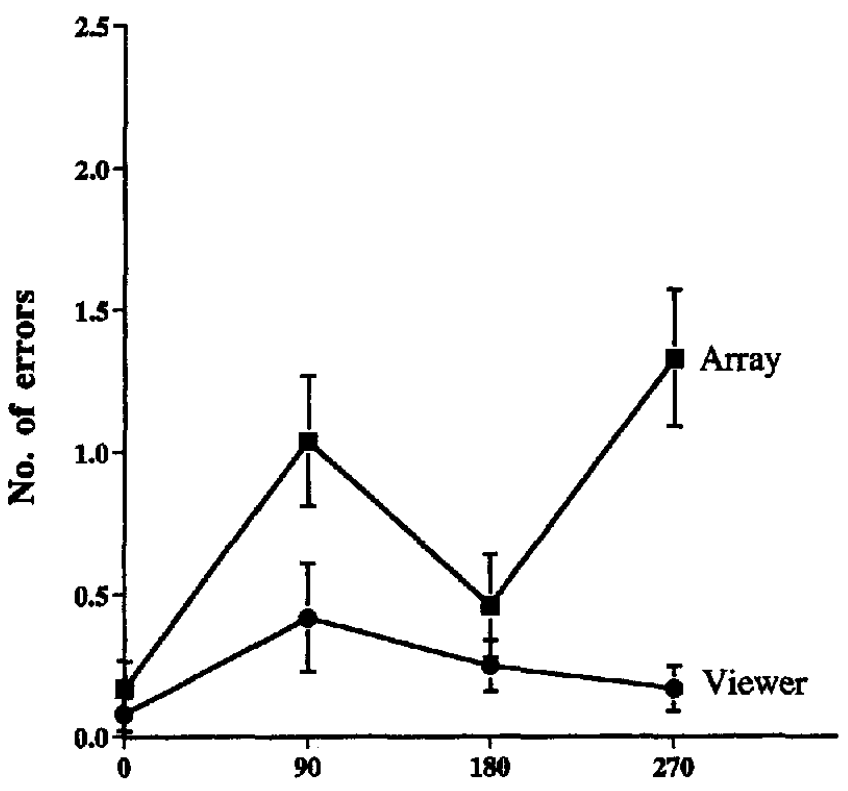

Degree of rotation

Figure 3. Mean errors and standard errors for the 16 trials, Experiment 1.

errors in array $(M=0.68)$ versus viewer $(M=0.30)$ conditions, $p=.332$. We also calculated the mean proportion of egocentric errors for each task. ${ }^{3}$ Again, no difference was found between array $(M=0.18)$ and viewer $(M=0.17)$ conditions, $p=.933$.

\section{Position Analysis}

The ANOVAs performed assessed whether responses concerning the right/left and front/back positions of objects in the array affected RTs and error rates. Right/left responses $(M=2.31 \mathrm{~s})$ were significantly slower than top/bottom responses $(M=2.01 \mathrm{~s})$ for both array and viewer conditions, $F(1,20)=6.95, p<.016$. A similar trend was found with errors (right/left: $M=0.60$ error; top/bottom: $M=0.35$ error) for both array and viewer conditions, $F(1,16)=$ $18.67, p<.001$.

\section{Discussion}

As predicted, participants were more successful at updating locations of the array after imagining themselves rotating around the array than imagining the array itself rotating. These findings are similar to those of Presson (1982, Experiment 2).

For the viewer task, RTs exhibited the classic mental rotation function of increasing latencies up to $180^{\circ}$, with a dropoff beyond $180^{\circ}$ (Shepard \& Metzler, 1971). This suggests that participants imagined moving themselves around the array in the specified direction for $90^{\circ}$ and $180^{\circ}$ but imagined moving in the opposite direction for $270^{\circ}$ rotations. In contrast, response latencies in the array task continued to increase beyond $180^{\circ}$. There are several plausible interpretations of this finding, none of which can be made conclusively with the present data. For example, participants may have complied with the instruction to move in one direction because of the difficulty of the array task. Or, they might have been unable to use the reverse-direction strategy successfully.

The analysis of error underscores the difficulty of the array task. Significantly more errors were made in array, with the most occurring at $90^{\circ}$ and $270^{\circ}$. The dropoff in error for $180^{\circ}$ has been reported elsewhere (Presson, 1982) and is most probably due to participant's ability to reverse the positions of the objects in that condition. In contrast, the viewer task was performed with ease: Errors were few and were unrelated to the degree of rotation.

Differences in error patterns did not extend to egocentric errors. On average, participants made very few egocentric errors with item questions in both array and viewer tasks. These results replicate those of other studies with adults (Presson, 1982) and children (Huttenlocher \& Presson, 1979).

The analysis of RTs and errors as a function of specific positions in the array revealed poorer performance for responses to right/left positions versus top/bottom positions. This is in line with Franklin and Tversky's (1990) spatialframework model of space conceptualization, which posits that mental representations of space reflect the way the physical body interacts with the world. According to this view, the left/right axis is the least accessible axis of the body because it contains no asymmetries. Our results suggest that participants' representation of the array adhered to these constraints. This notion was explored further in Experiment 2.

\section{Experiment 2}

In Experiment 2, we examined whether altering the physical relationship between participants and the array would improve array performance. It is possible that having participants stand outside of the array of objects in Experiment 1 contributed to difficulties in performing the array task. In the present experiment, we placed participants in the center of the array so that the objects could be more easily encoded with respect to the egocentric frame. However, we predicted that performance in the viewer task would continue to be faster and less error-prone than in the array task.

The inside-array manipulation also allowed us to test further Tversky and colleagues' spatial-framework model of space conceptualization. They found that objects appearing behind participants in physical space were less accessible in mental space, compared with objects appearing before participants (Bryant, Tversky, \& Franklin, 1992; Franklin \& Tversky, 1990). We tested for a similar effect in the present experiment.

\footnotetext{
${ }^{3}$ For greatest accuracy, mean proportions in this and subsequent experiments were calculated only from errors that potentially could be egocentric. Therefore, errors for $0^{\circ}$ rotations were excluded from analysis.
} 


\section{Method}

\section{Participants}

Twenty-four University of Virginia undergraduates (11 women, 13 men) participated in the experiment as part of a research credit requirement. Two additional participants were excluded from the experiment for not following directions. All participents were tested individually; none knew of the hypothesis being tested.

\section{Materials}

The four-object array was used.

\section{Procedure}

The procedure was the same as Experiment 1 except that the observer stood in the center of the array, facing forward. Because of this setup, the four locations in the array were referred to as front, back, left, and right. Participants learned the item locations in a manner similar to that of Experiment 1, except that they were allowed to look over their shoulders to view the back object; they were not allowed to turn around. For the viewer task, participants imagined themselves rotating in place; in the array task, they imagined the array rotating around themselves.

\section{Results}

Like Experiment 1, the principal finding was that observers were faster and made fewer errors in the viewer task compared with the array task. 4

\section{Latency}

Figure 4 shows mean RTs and standard errors for correct responses in each task as a function of degree of rotation.

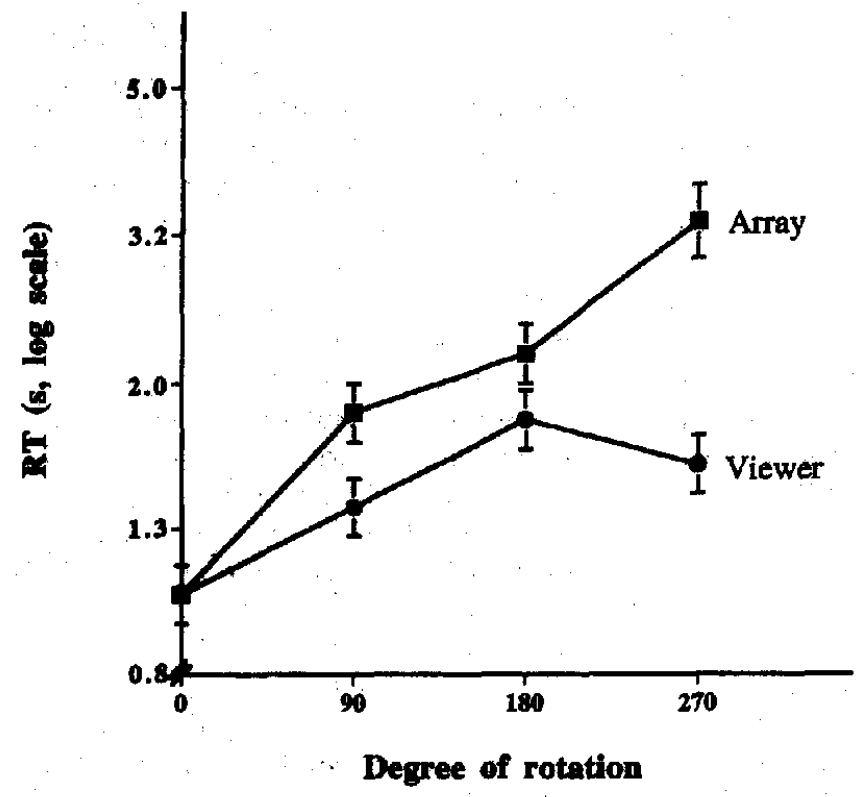

Figure 4. Mean reaction times (RT) and standard errors, Experiment 2. Values are in seconds, depicted in log space.

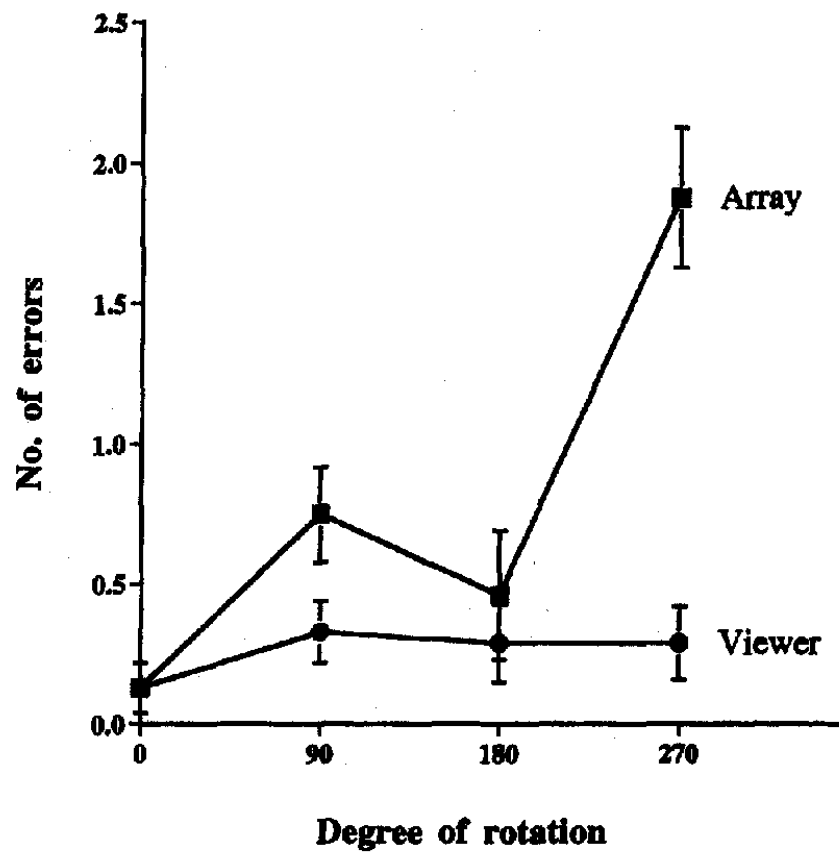

Figure 5. Mean errors and standard errors for 16 trials, Experiment 2.

Overall RT in the viewer condition ( $M=1.57 \mathrm{~s}$ ) was faster than in the array condition $(M=2.34 \mathrm{~s})$. The ANOVA performed on the mean scores yielded main effects of task, $F(1,16)=29.39, p<.001$, and degree, $F(3,48)=55.96$, $p<.0001$, and a significant Task $\times$ Degree interaction, $F(3,48)=13.41, p<.0001$. As in Experiment 1, contrast comparisons revealed that this interaction was a result of different functions of RT across degrees for each task (see Figure 4). In the array task, RT increased from $0^{\circ}$ to $90^{\circ}$ and $180^{\circ}$ to $270^{\circ}$, whereas in the viewer task, RT increased to $180^{\circ}$ but then decreased at $270^{\circ}$.

\section{Accuracy}

As in Experiment 1, participants were more accurate in the viewer condition ( $M=0.26$ errors) than in the array condition ( $M=0.80$ errors). Figure 5 shows mean errors and standard errors as a function of task and degree of rotation. The ANOVA performed on number of errors yielded main effects of task, $F(1,16)=12.37, p<.003$, and degree, $F(3,48)=16.48, p<.0001$, and a significant Task $\times$ Degree interaction, $F(3,48)=13.03, p<.0001$. Similar to the results of Experiment 1, the Task $\times$ Degree interaction revealed an increase in array errors for $90^{\circ}$ and $270^{\circ}$ but no effect of degree in the viewer condition.

In assessing egocentric errors across tasks, we first calculated the average egocentric errors of participants who

${ }^{4}$ An additional 2 (experiment) $\times 2$ (task) $\times 2$ (degree) ANOVA performed on the combined latency data from Experiments 1 and 2 revealed no difference between experiments, $F(1,46)=2.67$, $p<.11$. 
made errors in each task. As expected, no difference was found for number of egocentric errors in the array $(M=0.77)$ versus viewer $(M=.39)$ condition, $p=.289$. There was also no difference in the proportion of egocentric errors made in each task (array $M=0.22$; viewer $M=0.14$ ), $p=.452$.

\section{Position Analysis}

The ANOVAs performed assessed whether responses concerning the right/left and front/back positions of objects in the array affected RTs and error rates. Right/left responses $(M=1.81 \mathrm{~s})$ were slightly, but not significantly, slower than front/back responses $(M=1.65 \mathrm{~s})$ for array and viewer conditions, $p=.191$. Post hoc analyses yielded no difference between top and bottom responses, $p=.950$. For errors, the ANOVA revealed no overall effect of position but a significant Task $\times$ Position interaction, $F(1,16)=8.6$, $p<.01$. More right/left errors $(M=0.98$ error) were made than front/back errors $(M=0.63$ error) in the array condition, $F(1,23)=7.82, p<.01$, but not in the viewer condition, $p=.48$.

\section{Discussion}

As predicted, performance in the viewer task was faster and more accurate than performance in the array task, despite the fact that participants were positioned inside the array. Moreover, no difference was found between the results of Experiments 1 and 2. These results collectively extend Presson's (1982) item-question findings to observer placement both inside and outside of a large-scale array.

RTs in the viewer task continued to exhibit the classic mental rotation function, suggesting that participants had implemented reverse-rotation strategies for the $270^{\circ}$ case. In contrast, the increase in RTs for $270^{\circ}$ found in the array task indicated that, like Experiment 1, the reverse-rotation strategy was either inaccessible or unsuccessful in that task.

In Experiment 1, we found slower performance for responses to right/left positions versus top/bottom positions, in line with Franklin and Tversky's (1990) spatialframework model of space conceptualization. In the present experiment, that difference was diminished in all conditions except array errors. Moreover, contrary to the spatialframework model, we found no difference in performance for objects appearing behind, versus in front of, the observer. However, the absence of the latter front/back effect is likely due to the relative simplicity of our array. We used four objects arranged symmetrically about the observer, whereas Tversky and colleagues used between five and eight objects, which were distributed asymmetrically about the observer. The absence of a front/back effect in regular versus irregular arrays has been reported elsewhere by Easton and Sholl (1995), who attributed the finding to the relative ease with which regular arrays are learned.

\section{Experiment 3}

The results of the first two experiments suggest an advantage for updating object arrays after imagined viewer versus object rotations. However, Presson (1982) found that simply rephrasing the question slightly, from an emphasis on updating a position in the array to updating a single object, resulted in the reverse effect. That is, when participants were given a specific target and asked to determine its position in the array (referred to by Presson as a position question, e.g., "rotate yourself/table $180^{\circ}$, where's the car?"), they showed an advantage for imagined array rotations over viewer ones. This finding contradicts the hypothesis that poor array performance could be attributable to difficulties in the cognitive system's representation of the transforming objectrelative frame because the components of such frame do not differ across item and position experiments.

However, as Presson noted, participants may have been able to use a different strategy in the array position task. He speculated that they had solved the array position task by transforming single objects sequentially, rather than performing rotations of the entire array. For example, in solving the example above, participants could simply move the car $180^{\circ}$ while ignoring the rest of the array. In the viewer task, however, the entire array had to be updated. The implication here is that, in the array task, participants may not have actually performed rotations at all because the imagined displacement of the named object could have been performed via translation rather than rotation. Participants could simply translate the named object to its prescribed position with a motion similar to that of a single Ferris wheel car, which traverses a circular path without rotating. Imagined translations are generally thought to be easier than imagined rotations (Easton \& Sholl, 1995; Rieser, 1989). Thus, poor viewer performance with position questions was possibly due to an unintentional confound in the corresponding array task.

In Experiment 3, we used a twofold approach to examine these issues. First, we were interested in possible effects of question format on rotation task performance. Participants made imagined viewer and array rotations in response to position questions. We predicted that performance in the position-question array task would improve over previous array findings with item questions. Second, we investigated whether participants were performing holistic rotations in the array task. We accomplished this by randomly adding catch trials into both tasks. The catch trials required participants to supply the position of any object in the array from a newly imagined array/viewer viewpoint (arrived at by answering a position question). For example, if the position question given was "Rotate yourself/array $180^{\circ}$, where's the car?" the catch-trial question might be "Now, what's on your right?" We expected the catch-trial response latencies, generally, to be slower than position-question latencies for both array and viewer tasks because of the increased mental load elicited by a second updating question. However, if participants in the array task did not initially perform an imagined rotation of the entire array during the position question, answering the catch trial would be much more difficult. Thus, we predicted that performance in the array task would show marked impairment on the catch questions compared with that of the viewer task. 


\section{Method}

\section{Participants}

Twenty-four University of Virginia undergraduates (13 women, 11 mon) participated in the experiment as part of a research credit requinament. All were tested individually; none knew of the hypothesis being tested.

\section{Materials}

The four-object array was used.

\section{Procedure}

The procedure was exactly the same as in Experiment 1, except for the question format. For each trial, participants received the degree of rotation followed by an object in the array (position question), for example " $180^{\circ}$, where's the car?" They responded by naming a position in the array. They were instructed to answer as quickly and accurately as possible. Four catch questions were also included within each task. A catch trial always occurred after a given position question. For each catch trial, the participant was given a position in the array and responded by naming the object that corresponded to that position after the imagined rotation from the first (position) question. For example, a questioning sequence might be, " $180^{\circ}$, where's the car?" [participant gives first answer]; "Now, what's on the left?" [participant gives second answer]. The catch trials were explained to participants before testing; however, they had no advanced waming of the occurrence of a given catch trial once testing began.

\section{Design}

The design was identical to that of Experiment 1, except for the addition of the catch trials. In each task, four catch trials were quasirandomly interspersed after the position questions. Each location tested (i.e., top, bottom, right, left) was randomly paired once with one of the position questions' four degrees of rotation (i.e., $0^{\circ}, 90^{\circ}, 180^{\circ}, 270^{\circ}$ ), with the result that the four catch trials collectively contained all four locations and degrees of rotation.

\section{Results}

\section{Position Question: "Where's the Car?"}

The principal finding was again that performance in the viewer task was faster and more accurate than in the array task. However, a comparison of the results with those of Experiment 1 revealed that array RTs and errors in the present experiment were reduced compared with Experiment 1, whereas viewer RTs and errors were not.

Latency. Figure 6 shows mean scores and standard errors for correct responses in each task as a function of degree of rotation. ${ }^{5}$ Overall performance in the viewer task $(M=1.42 \mathrm{~s})$ was slightly faster than the array task $(M=1.59 \mathrm{~s})$. The ANOVA revealed main effects of sex, $F(1,20)=4.98, p<.04$; task, $F(1,20)=6.05, p<.03$; and degree, $F(3,60)=45.18, p<.0001$, and a significant Task $\times$ Degree interaction, $F(3,60)=6.85, p<.0001$. The effect of sex was due to the fact that men responded faster than women in both array (men: $M=0.12 \mathrm{~s}$; women: $M=0.19 \mathrm{~s}$ ) and viewer (men: $M=0.03 \mathrm{~s}$; women: $M=0.16$



Figure 6. Mean reaction times (RT) and standard errors for position questions, Experiment 3. Values are in seconds, depicted in log space.

s) conditions. As is evidenced in Figure 6, the task effect was clearly driven by the $270^{\circ}$ rotation condition. A secondary ANOVA with the $270^{\circ}$ rotation removed confirmed this fact, yielding no effect of task, $F(1,20)=0.16, p<.7 .^{6}$ Contrast comparisons revealed patterns similar to the first two experiments. For the array task, RT increased at $90^{\circ}$ compared with $0^{\circ}$ and at $270^{\circ}$ compared with $180^{\circ}$. For the viewer task, RT was greater at $90^{\circ}$ compared with $0^{\circ}$; however, there was no difference between $90^{\circ}, 180^{\circ}$, and $270^{\circ}$.

To assess how RT improved with position questions versus item questions, the data of Experiments 1 and 3 were compared. Overall, improvement was greater for array than viewer tasks across the two experiments. A2 (experiment) $\times$ 2 (task) $\times 4$ (degree) mixed-design ANOVA revealed main effects of experiment, $F(1,46)=17.79, p<.0001$; task, $F(1,46)=36.21, p<.0001 ;$ and degree, $F(3,138)=94.05$, $p<.0001$. A significant Task $\times$ Degree interaction was reproduced, $F(3,138)=22.74, p<.0001$. More importantly, a significant Experiment $\times$ Task interaction was found, $F(1,46)=11.22, p<.002$. With Bonferroni-type adjustment, simple comparisons revealed a significant reduction of response latency from Experiment 1 to 3 for the array,

\footnotetext{
${ }^{5}$ Mean RTs that were greater than three standard deviations above the group mean of a given array or viewer degree condition were replaced with the group condition mean. In Experiment 2, one array RT and one viewer RT were replaced.

6 Similar analyses performed on the data of Experiments 1, 3, and 4 yielded a different result: With the $270^{\circ}$ rotations removed, task effects were still significant.
} 
$F(1,46)=25.66, p<.0001$, task but not for the viewer task. The mean reduction in RT was much greater for the array $(M=1.38 \mathrm{~s})$ task than for the viewer task $(M=0.22 \mathrm{~s})$.

Accuracy. Although overall performance tended to be better in the viewer task ( $M=0.17$ errors) than in the array task ( $M=0.30$ errors), this difference did not reach significance. Figure 7 shows mean errors and standard errors as a function of task and degree of rotation. The ANOVA performed on the error data revealed no main effects or significant interactions.

To assess how accuracy improved with position questions versus item questions, the error data of Experiments 1 and 3 were compared. Overall, improvement was greater for the array task than the viewer task across the two experiments. A 2 (experiment) $\times 2$ (task) $\times 4$ (degree) mixed-design ANOVA revealed main effects of experiment, $F(1,46)=$ $6.25, p<.016$; task, $F(1,46)=20.41, p<.0001$; and degree, $F(3,138)=11.99, p<.0001$. A significant Task $\times$ Degree interaction was reproduced, $F(3,138)=7.54, p<$ .0001 . Most notably, the Experiment $\times$ Task interaction was also significant, $F(1,46)=5.85, p<.020$. Simple comparisons with Bonferroni-type adjustment revealed a significant reduction in errors from Experiment 1 to 3 for array, $F(1,46)=9.43, p<.004$, but not viewer $(p=.464)$ tasks.

In assessing egocentric errors across tasks, we first calculated the average egocentric errors of participants who made errors in each task. No difference was found for number of egocentric errors in array $(M=0.31)$ versus viewer $(M=0.30)$ tasks, $p=.956$. Although the proportion of egocentric errors in array $(M=0.12)$ was less than in viewer $(M=0.25)$ conditions, the difference did not reach significance, $p=.339$.



Degree of rotation

Figure 7. Mean errors and standard errors for position questions (16 trials), Experiment 3.

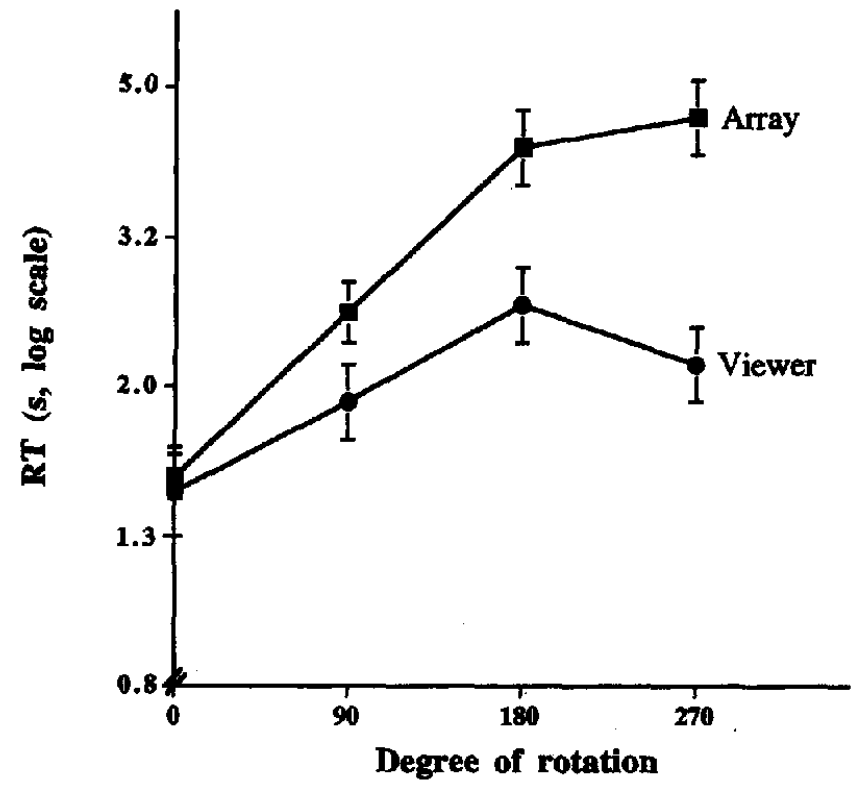

Figure 8. Mean reaction times (RT) and standard errors for catch trials, Experiment 3. Values are in seconds, depicted in log space. Degree here refers to the amount of rotation traversed in the position question.

Position analysis. Because responses corresponded to objects in the array rather than their positions, position analyses were not conducted.

\section{Catch Question: "What's on the Left?"}

The principal finding was that performance was faster in the viewer than in the array condition.

Latency. Figure 8 shows mean scores and standard errors for correct responses, as a function of degree. Degree here refers to the amount of imagined rotation for the position question because participants did not imagine another rotation for the catch trial. As expected, catch-trial RTs were slower than position-question RTs, and their function was steeper (cf. Figure 6), most likely because of the additional mental effort they elicited. Overall, responses to the catch questions were faster in viewer $(M=2.39 \mathrm{~s})$ than in array $(M=3.59 \mathrm{~s})$ conditions. The ANOVA revealed main effects of task, $F(1,20)=11.05 ; p<.003$, and degree, $F(3,60)=22.02, p<.001$, and a significant Task $\times$ Degree interaction, $F(3,60)=5.83, p<.001$. Contrast comparisons indicated that for the array task, RT increased for position-question rotations of $90^{\circ}$ compared with $0^{\circ}$ $(p<.001)$, and from $180^{\circ}$ compared with $90^{\circ}(p<.01)$ but did not increase further at position-question rotations of $270^{\circ}$. In the viewer task, RTs did not differ as a function of position-question degrees of rotation.

Accuracy. Because there was only one catch trial per degree for a total of four trials, a paired $t$ test was performed on the mean error for each task. This analysis revealed significantly fewer errors in the viewer task $(M=0.42)$ than in the array task $(M=0.92), t(23)=-3.13, p<.005$. 


\section{Discussion}

Similar to the results of Presson (1982), changing the test question from one of updating the array given a position (Experiment 1) to one of updating given a specific object (Experiment 3) resulted in improved performance for the array task. In contrast, as assessed by between-experiment comparisons, viewer performance was essentially unchanged across question formats. How do we account for these findings? We proposed that the position-question format would allow participants to make imagined translations of single abjects instead of rotations of the entire array in the array task. On the other hand, the viewer task in Experiment 2 would still require rotations of the entire array.

The results of the catch-trial questions supported this claim. The general difficulties found in the array task versus the viewer task, as evidenced in both greater RT and number of errors, indicated that participants in the array task had problems keeping track of the position of more than one element in the array during the position-question transformation. In contrast, the viewer catch trials were performed with relative ease, suggesting that participants had updated the entire array for position questions. These findings imply that position questions (e.g., "Where is the car?") are inadequate for assessing performance of imagined array rotations because they lead to a spurious improvement in array updating.

The pattern of results found for viewer position-question RTs was interesting because it did not exhibit the classic mental rotation function. That is, latencies for rotations beyond $90^{\circ}$ were essentially the same. This finding suggests, at first glance, that participants were able to instantaneously transport themselves to all locations of the array, regardless of a given location's physical distance. Kosslyn (1994) termed this a blink transformation. However, it is possible that participants used a symmetry-reversal strategy for the $180^{\circ}$ rotation, which reduced response time to that of the $90^{\circ}$ and $270^{\circ}$ (i.e., $90^{\circ}$ in the opposite direction) rotations.

Another noteworthy finding was that the amount of egocentric errors participants made did not differ across array and viewer tasks. This finding contradicts previous studies, which reported more egocentric errors in viewer position questions for both adults (Presson, 1982) and children (Huttenlocher \& Presson, 1979). Moreover, the current finding does not fit with Huttenlocher and Presson's notion of primary and secondary reference-frame conflicts. This hypothesis predicts that position questions should result in more egocentric errors in the viewer task because of the mismatch between perceived and imagined relations to the environmental reference frame.

Finally, an effect of sex was found for position-question latencies. Men performed faster than women for both array and viewer tasks. This finding is in line with several studies showing superior performance in men for certain spatial tasks (e.g., Linn, \& Petersen, 1985; Macoby \& Jacklin, 1974).

\section{Experiment 4}

The findings of the first three experiments suggest that it is more difficult to imagine the rotation of an array of objects than to imagine the rotation of the self around the array. We next sought to ascertain the conditions under which performance with imagined object rotations could be improved. According to our hypothesis, difficulties in the array task arise from participants' difficulties in performing cohesive rotations of the array's object-relative reference frame. In Experiment 4, we collapsed the array into one object: a block with four differently colored edges. In this way, the four components to be learned were spatially adjoined and thus more likely to be encoded as a single unit, similar to the order of the four axes of the body used in the viewer task. Huttenlocher and Presson (1979) tested children with a similar manipulation and found a viewer advantage; however, they did not examine whether performance in either task improved across four- and one-object configurations.

In the present study, participants performed in two conditions. For the object task, they made imagined rotations of the block; for the viewer task, they imagined themselves rotating around the block. We predicted that performance in the object task would improve with respect to viewer performance.

\section{Method}

\section{Participants}

Twenty-four University of Virginia undergraduates (13 women, 11 men) participated in the experiment as part of a research credit requirement. Two additional participants were excluded from the experiment for having more than a $50 \%$ error rate in the object task. All participants were tested individually; none knew of the hypothesis being tested.

\section{Materials}

The wooden block was used (see Object Materials, General Method). It was placed on one of the pedestals from the array.

\section{Procedure}

The procedure was identical to Experiment 1 except that imagined rotations were performed with respect to a single object rather than an array of four objects.

\section{Results}

The principal finding was again that performance was faster and more accurate in the viewer task than in the object task.

\section{Latency}

Figure 9 shows mean scores and standard errors for correct responses in each task as a function of degree of rotation. ${ }^{7}$ Participants responded more quickly in the viewer

\footnotetext{
${ }^{7}$ Mean RTs that were greater than three standard deviations above the overall mean of a given array or viewer degree condition were replaced with the group condition mean. In Experiment 4, five array RTs and one viewer RT were replaced.
} 




Figure 9. Mean reaction times (RT) and standard errors, Experiment 4 . Values are in seconds, depicted in log space.

task $(M=1.70 \mathrm{~s})$ than in the object task $(M=3.48 \mathrm{~s})$. The ANOVA revealed main effects of task, $F(1,16)=42.84$, $p<.0001$, and degree, $F(3,48)=71.94, p<.0001$, and a significant Task $\times$ Degree interaction, $F(3,48)=27.68$, $p<.0001$. We found the same RT pattem as in the other experiments. For the object task, RT increased at $90^{\circ}$ compared with $0^{\circ}$ and at $270^{\circ}$ compared with $180^{\circ}$, but there was no difference between $90^{\circ}$ and $180^{\circ}$. For the viewer task, RT was greater at $90^{\circ}$ compared with $0^{\circ}$; however, there was no difference between $90^{\circ}, 180^{\circ}$, and $270^{\circ}$.

To assess whether RT improved with rotations of the array versus block, the data of Experiments 1 and 4 were compared. No improvement was found, for either task. A 2 (experiment) $\times 2$ (task) $\times 4$ (degree) mixed-design ANOVA revealed main effects of task, $F(1,46)=81.49, p<.0001$, and degree, $F(3,138)=55.13, p<.0001$, but no effect of experiment $(p=.868)$ nor significant interactions.

\section{Accuracy}

Participants were more accurate in the viewer task ( $M=0.31$ errors) compared with the object task $(M=0.90$ errors). Figure 10 shows mean errors and standard errors by degree of rotation. The ANOVA performed on the errors revealed main effects of task, $F(1,16)=28.32, p<.0001$, and degree, $F(3,48)=11.98, p<.0001$, and a significant Task $\times$ Degree interaction, $F(3,48)=6.05, p<.001$. In the object task, errors were greater at $90^{\circ}$ than at $0^{\circ}$ and at $270^{\circ}$ compared with $180^{\circ}$, with no difference between $90^{\circ}$ and $180^{\circ}$. In the viewer task, errors increased at $90^{\circ}$ compared with $0^{\circ}$ but did not differ between $90^{\circ}, 180^{\circ}$, and $270^{\circ}$.

To assess whether accuracy improved with rotations of the array versus block, the data of Experiments 1 and 4 were compared. No improvement was found, for either task. A 2 (experiment) $\times 2$ (task) $\times 4$ (degree) mixed-design ANOVA revealed main effects of task, $F(1,46)=41.12, p<.0001$, and degree, $F(3,138)=21.17, p<.0001$, but no effect of experiment $(p=.258)$ nor significant interactions.

In assessing egocentric errors across tasks, we first calculated the average egocentric errors of participants who made errors in each task. This yielded the same value for both array $(M=0.63)$ and viewer $(M=0.63)$ tasks. However, the proportion of egocentric errors made in the array task $(M=0.13)$ was significantly less than in the viewer task $(M=0.48), t(38)=7.45, p=.01$.

\section{Position Analysis}

The ANOVAs performed assessed whether responses concerning the right/left and top/bottom positions of the object's parts affected RTs and error rates. Right/left responses $(M=2.38 \mathrm{~s})$ were significantly slower than top/ bottom responses $(M=2.04 \mathrm{~s})$ for array and viewer tasks, $F(1,20)=9.42, p<.006$. For errors, the ANOVA revealed no overall effect of position, $p<.50$.

\section{Discussion}

The collapse of the array into one object did not facilitate object performance. Participants took longer to respond and made more errors in the object task than in the viewer task, in a manner similar to the array performance of Experiment 1. RT performance in the object task also produced a rotation function similar to that of the array task of Experiment 1, increasing RTs beyond $180^{\circ}$. These results suggest that it remains difficult to predict the cohesive rotation of several

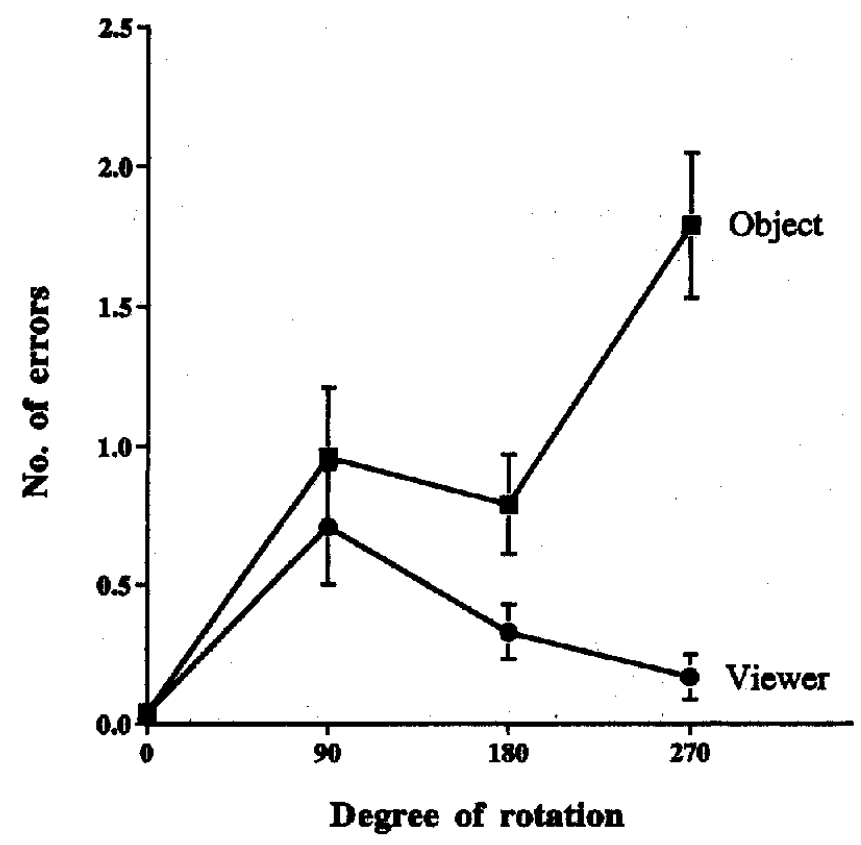

Figure 10. Mean errors and standard errors for the 16 trials, Experiment 4. 
components even when they are spatially connected as a single object.

The pattern of RT responses found in the viewer task was identical to that of Experiment 3, with no difference beyond rotations of $90^{\circ}$. This finding is not in accord with the classic reaction time function and suggests that participants used either blink transformations or short-cut strategies.

Although the number of egocentric errors made by participants was similar across array and viewer tasks, they accounted for a significantly greater percentage of errors in viewer than in array tasks ( $48 \%$ vs. $13 \%$, respectively). This unexpected finding contradicts those of Experiments 1 and 2 , which also used item questions, as well as the results of previous studies using item questions (Huttenlocher \& Presson, 1979; Presson, 1982).

Finally, the position analysis revealed poorer performance for responses to left/right positions in the array versus top/bottom positions. This replicates the finding of Experiment 1 and indicates that the mental construction of space is similar for arrays and single objects.

\section{Experiment 5}

Introduction of the block in Experiment 4 was evidently not enough to facilitate imagined transformations of a cohesive object. To continue testing our hypothesis, we next attempted to provide participants with additional configural information about the rotation object. Huttenlocher and Presson (1979) found that using a telephone as the array/ object resulted in improved array rotation performance for children. Interestingly, the improvement occurred only when the telephone was visible during the imagined rotation trials; when it was out of sight, a typical viewer advantage prevailed. We tried a similar manipulation in Experiment 5 by replacing the block with a toy car. The car had four spatially connected components: hood, trunk, driver side, and passenger side. Its configuration was thus both encodable as a single unit and highly familiar.

Like Experiment 4, participants answered questions about the position of a given component of the car after making both imagined viewer and object rotations. We predicted that performance would improve in the object task to nearviewer levels.

\section{Method}

\section{Participants}

Twenty-four University of Virginia undergraduates (13 women, 11 men) participated in the experiment as part of a research credit requirement. Four additional participants were excluded from the experiment, two for not following directions, and two for having more than a $50 \%$ error rate in the object task. All participants were tested individually; none knew of the hypothesis being tested.

\section{Materials}

The car was placed on an individual pedestal; as in Experiment 4 (see Figure 11). top

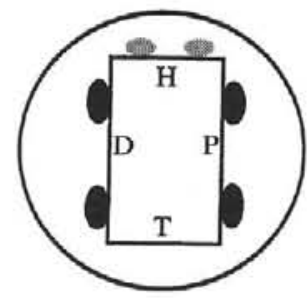

left



right

bottom

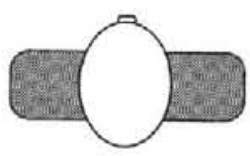

Figure 11. Insert, top right: A close-up overhead view of the car, Experiment 5. $\mathbf{H}=$ hood; $\mathrm{P}=$ passenger's side; $\mathrm{T}=$ trunk; and $\mathrm{D}=$ driver's side. Main figure: Overhead view of one orientation of the car with respect to the positions in the array. In the three other orientations, $\mathbf{H}$ pointed to the right, bottom, and left, respectively.

\section{Procedure and Design}

The procedure and design were identical to those of Experiment 4, except that participants learned the positions of the components of the car.

\section{Results}

The principal finding was that performance in the viewer task was faster and more accurate than in the object task.

\section{Latency}

Mean scores and standard errors for correct responses in each task as a function of degree of rotation are shown in Figure $12 .{ }^{8}$ Participants responded more quickly in the viewer task $(M=1.82 \mathrm{~s})$ than in the object task $(M=2.66 \mathrm{~s})$. The ANOVA performed on mean scores yielded main effects of task, $F(1,16)=17.98, p<.001$, and degree, $F(3,48)=$ $26.72, p<.0001$, and a significant Task $\times$ Degree interaction, $F(3,48)=10.4, p<.0001$. For the object task, contrast comparisons revealed longer latencies for $90^{\circ}$ compared with $0^{\circ}$ and for $270^{\circ}$ compared with $180^{\circ}$. However, there was no difference between $90^{\circ}$ and $180^{\circ}$. For the viewer task, RT increased from $0^{\circ}$ to $90^{\circ}$ only; there was no difference between $90^{\circ}, 180^{\circ}$, and $270^{\circ}$.

\footnotetext{
${ }^{8}$ Mean RTs that were greater than three standard deviations above the overall mean of a given array or viewer degree condition were replaced with the group condition mean. In Experiment 3, one array RT and one viewer RT were replaced.
} 
Separate ANOVAs performed on the RT scores of each task as a function of the four possible orientations of the car revealed no orientation effects for either the object task $(p=.240)$ or the viewer task $(p=.681)$.

To assess how RT improved with rotations of the car versus block, the data of Experiments 4 and 5 were compared. Overall, RT improved for the object task but not for the viewer task across the two experiments. A 2 (experiment) $\times 2$ (task) $\times 4$ (degree) mixed-design ANOVA revealed main effects of task, $F(1,46)=57.00, p<.0001$, and degree, $F(3,138)=55.13, p<.0001$. A significant Task $\times$ Degree interaction was reproduced, $F(3,138)=$ $35.96, p<.0001$. More importantly, a significant Experiment $\times$ Task interaction was found, $F(1,46)=6.95, p<$ .011. Simple comparisons revealed a significant reduction of response latency from Experiment 4 to 5 for the object task, $F(1,46)=4.09, p<.049$, but not for the viewer task $(p=.767)$. A significant Experiment $\times$ Degree interaction was also found, $F(3,138)=4.10, p<.008$, which was attributable solely to a reduction from Experiment 4 to 5 of RTs for $270^{\circ}, F(1,46)=4.14, p<.048$.

\section{Accuracy}

Participants were generally more accurate in the viewer task ( $M=0.44$ errors) than in the object task $(M=0.84$ errors). Figure 13 shows mean errors and standard errors by degree of rotation. The ANOVA performed on the errors yielded main effects of task, $F(1,16)=4.38, p<.05$, and degree, $F(3,48)=10.92, p<.0001$, and a significant Task $\times$ Degree interaction, $F(3,48)=4.24, p<.010$. Simple comparisons revealed that object errors were greater at $90^{\circ}$ compared with $0^{\circ}$ and at $270^{\circ}$ compared with $180^{\circ}$, but there was no difference between $90^{\circ}$ and $180^{\circ}$. For the

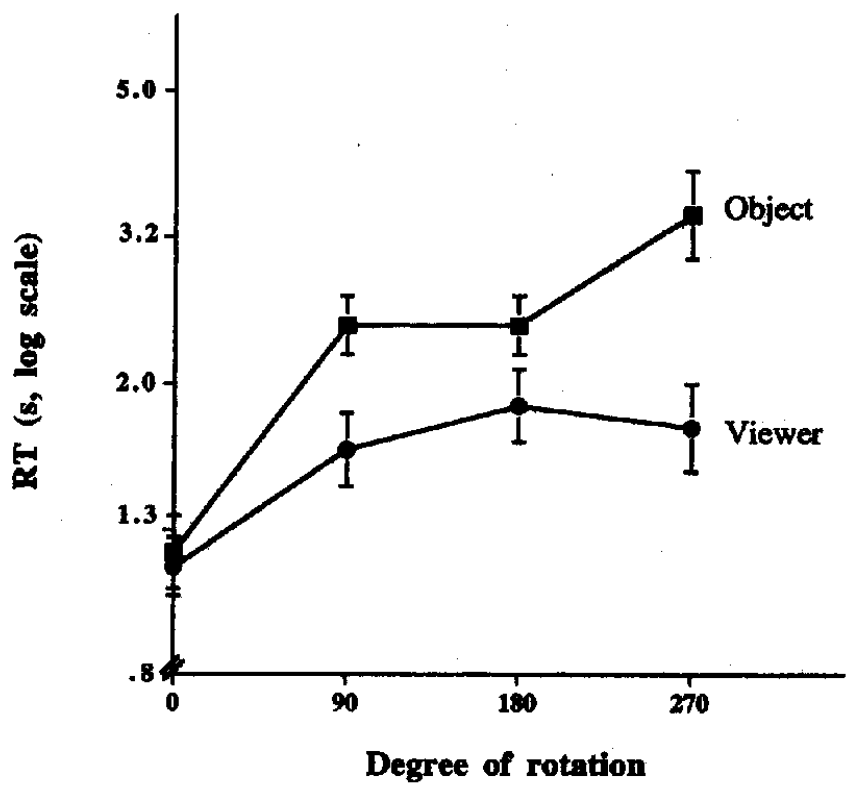

Figure 12. Mean reaction times (RT) and standard errors, Experiment 5 . Values are in seconds, depicted in log space.

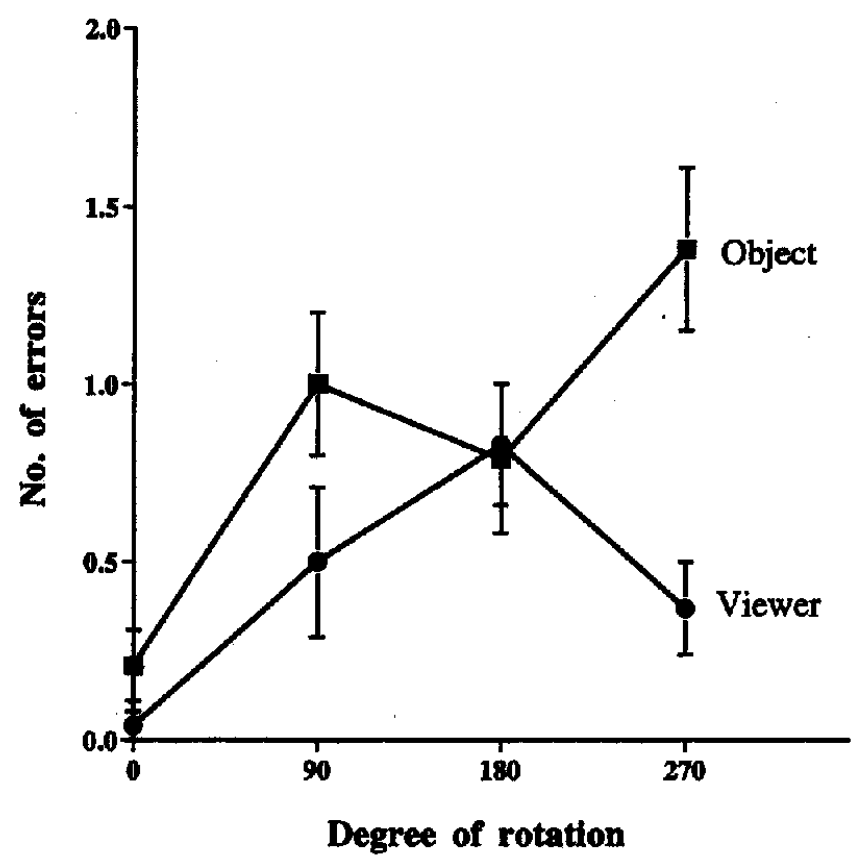

Figure 13. Mean errors and standard errors for the 16 trials, Experiment 5.

viewer task, errors increased at $90^{\circ}$ compared with $0^{\circ}$ and decreased at $270^{\circ}$ compared with $180^{\circ}$. Errors did not differ between $90^{\circ}$ and $180^{\circ}$.

Separate ANOVAs performed on the errors of each task as a function of the four possible orientations of the car revealed no orientation effects for either the object task $(p=.487)$ or the viewer task $(p=.676)$.

To assess whether accuracy improved with rotations of the car versus block, the data of Experiments 4 and 5 were compared. No improvement was found across experiments; both were highly accurate. A 2 (experiment) $\times 2$ (task) $\times 4$ (degree) mixed-design ANOVA revealed main effects of task, $F(1,46)=20.96, p<.0001$, and degree, $F(3,138)=$ $17.79, p<.0001$, but no effect of experiment $(p=.128)$ nor significant interactions.

In assessing egocentric errors across tasks, we first calculated the average egocentric errors of participants who made errors in each task. This yielded similar values for both array $(M=0.71)$ and viewer $(M=0.94)$ tasks, $p=.487$. However, the proportion of egocentric errors in the array task $(M=0.15)$ was again significantly less than in the viewer task $(M=0.44), t(36)=8.04, p=.007$.

\section{Position Analysis}

The ANOVAs performed assessed whether responses concerning the right/left and top/bottom positions of the object's parts affected RTs and error rates. Right/left responses $(M=2.24 \mathrm{~s})$ were significantly slower than top/ bottom responses $(M=1.94 \mathrm{~s})$ for array and viewer tasks, $F(1,20)=6.46, p<.019$. For errors, the ANOVA revealed no overall effect of position, $p<.80$. 


\section{Discussion}

Despite the introduction of the car, performance in the viewer task continued to be faster and more accurate than in the object task. However, our hypothesis was partially supported. Although replacing the block with the car did not improve object performance to the level of viewer, it did improve it with respect to the object-block task of Experiment 4. A comparison of the RT data of both experiments revealed a significant reduction from object-block to objectcar, but no difference between the corresponding viewer tasks. These results are consistent with those of Huttenlocher and Presson's (1979) telephone study with children, although we found improved object performance even though the car was not visible. More importantly, these findings lend support to the notion that poor object-rotation performance stems from observers' inherent problems with imagining a cohesive representation of the object-relative frame during transformation. By our account, performance in the object task improved from block to car because participants were better able to maintain the relationship among parts of the car during imagined rotation. An alternative explanation might be that the improvement was due to a general reduction in memory load. However, this argument is undermined by the fact that performance in the corresponding viewer tasks was equivalent across experiments.

Similar to Experiment 4, the number of egocentric errors made by participants in the present study accounted for a significantly greater percentage of errors in the viewer task than in the array task ( $44 \%$ vs. $15 \%$, respectively). In contrast, Experiments 1 and 2, which also tested item questions, found no task difference for egocentric errors (e.g., Experiment 1: viewer, 17\%; array, 18\%). How can we account for this dissociation? The biggest difference between experiments is that the earlier ones ( 1 and 2 ) involved imagined self-transformations about a large array of objects, whereas the later ones (4 and 5) involved imagined selftransformations about a small object. Perhaps the relatively smaller distances between components of the object, as compared with the array, contributed to a greater overlap of physical and projected reference frames on self-transformation. This issue warrants further empirical investigation.

\section{Experiment 6}

Experiment 5 's improved object performance with the car suggests that imagined object rotations can be facilitated if the relationship among components can be maintained more readily in the imagination. Experiment 6 explored this hypothesis further by incorporating a physical rotation into the design. Several studies have demonstrated that spatial updating during imagined self-rotation is improved when accompanied by a corresponding physical rotation (Farrell \& Roberteon, 1998; Hardwick, McIntyre, \& Pick, 1976; Presson \& Montello, 1994; Rieser, 1989; Rieser, Guth, \& Hill, 1986). For example, Rieser (1989; Rieser et al., 1986) found that participants took less time to point to objects after an imagined trensformation when they were actually guided to the new viewpoint compared with when they simply imagined moving to it. Thus; proprioceptive information about body position helped facilitate spatial updating when vision was not available.

In our last study, we applied this logic to the investigation of whether physical rotations of an object would affect updating in imagined object rotations. Participants performed both viewer and object tasks, using Experiment 4's block. However, the present object task included a passive rotation of the block in participants' hand as they performed each rotation trial (object task). The experimenter turned the block in such a way as to ensure a constant rotation speed across participants. This manipulation was not proprioceptive because it did not involve changes in body position. Rather, it provided haptic information specifying the object's orientation at every phase of the transformation. The viewer task was similar to that of Experiment 4, except that participants imagined rotating themselves around the block in their hand. We predicted that the physical turning of the block in the object task would significantly enhance participants' imagery of a cohesive object transformation and would thus further improve performance in the object task to the level of the viewer task.

\section{Method}

\section{Participants}

Forty-eight University of Virginia undergraduates (25 women, 23 men) participated in the experiment as part of a research credit requirement. Six additional participants were excluded from the experiment, 1 because of experimenter error and 5 for having more than a $50 \%$ error rate in the array task. ${ }^{9}$

\section{Materials}

The block was used.

\section{Procedure and Design}

Participants stood with their nondominant hand extended in front of them, palm-side up, while keeping their elbow touching the side of their body. The block was then placed in the participant's hand in one of four orientations. Participants memorized the positions of the colors on the block following the same procedure as Experiment 3. Imagined rotations were always performed in a clockwise direction. In the array task, the experimenter smoothly turned the block in participants' hand to the correct degree of rotation while the degree of rotation was announced. The velocity of the block turning was adjusted so that the end of the rotation coincided with the end of the announcement of degree. Like the previous experiments, RT was measured from the end of the experimenter's question (e.g., "What's on the right?") to the beginning of the participant's response. The block always remained fiat so that it was touching the participant's hand. Between each trial, the experimenter lifted the block off the hand to return it to its starting position to prevent additional haptic contact. In the viewer task, participants imagined rotating themselves around the hand-held block. A separate control group of 24 participants ran in both object and viewer tasks without the block-turning manipulation.

\footnotetext{
${ }^{9}$ Four of the 6 participants eliminated from the study were from the control group (see Procedure and Design).
} 


\section{Results}

Overall, performance on the object task improved compared with the previous experiments and was more similar to performance in the viewer task, although viewer performance remained superior to object performance.

\section{Latency}

Mean scores and standard errors of correct responses in each task as a function of degree of rotation are shown in Figure 14. Participants responded more quickly in the viewer $\operatorname{task}(M=1.43 \mathrm{~s})$ than in the object task $(M=1.90 \mathrm{~s})$. The ANOVA performed on mean scores yielded main effects of task, $F(1,16)=7.81, p<.011$, and degree, $F(3,60)=$ $17.64, p<.001 . .^{10}$ In addition, the analysis revealed a significant Task $\times$ Degree interaction, $F(3,60)=6.91, p<$ .001 , and Task $\times$ Task order interaction, $F(1,20)=4.51$, $p<.05$.

Several factors illustrate that object and viewer response latencies were much closer than in previous studies. First, although there was an overall effect of task condition, this effect appeared to be driven by the $270^{\circ}$ rotation. To assess this further, a subsequent 2 (task) $\times 2$ (task order) $\times 2$ (sex) $\times 4$ (degree) ANOVA with $270^{\circ}$ removed was performed. With $270^{\circ}$ removed from both tasks, the difference between object and viewer disappeared, $F(1,16)=$ $2.10, p=.16$.

Second, unlike the block-turning group, participants in the control group revealed response patterns similar to the previous experiments. RTs were much faster in the viewer task $(M=1.70 \mathrm{~s})$ than in the object task $(M=2.74 \mathrm{~s}), F(1$, 16) $=21.86, p<.001$. Moreover, a comparison of RTs in the control and block-turning object tasks revealed a signifi-

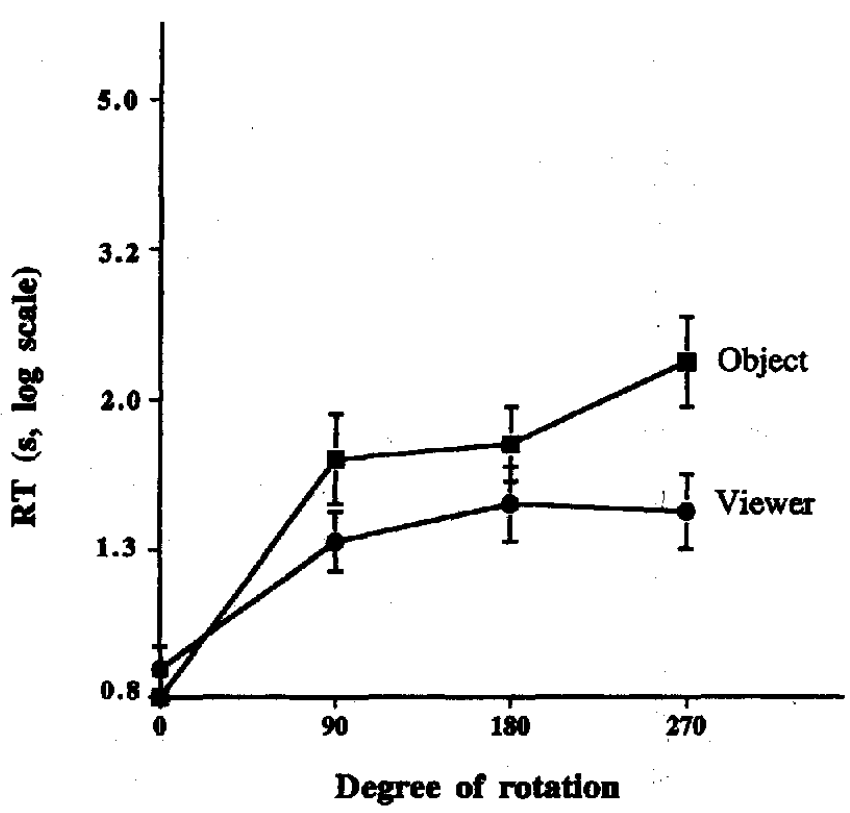

Figure 14. Mean reaction times (RT) and standard errors, Experiment 6 . Values are in seconds, depicted in log space.

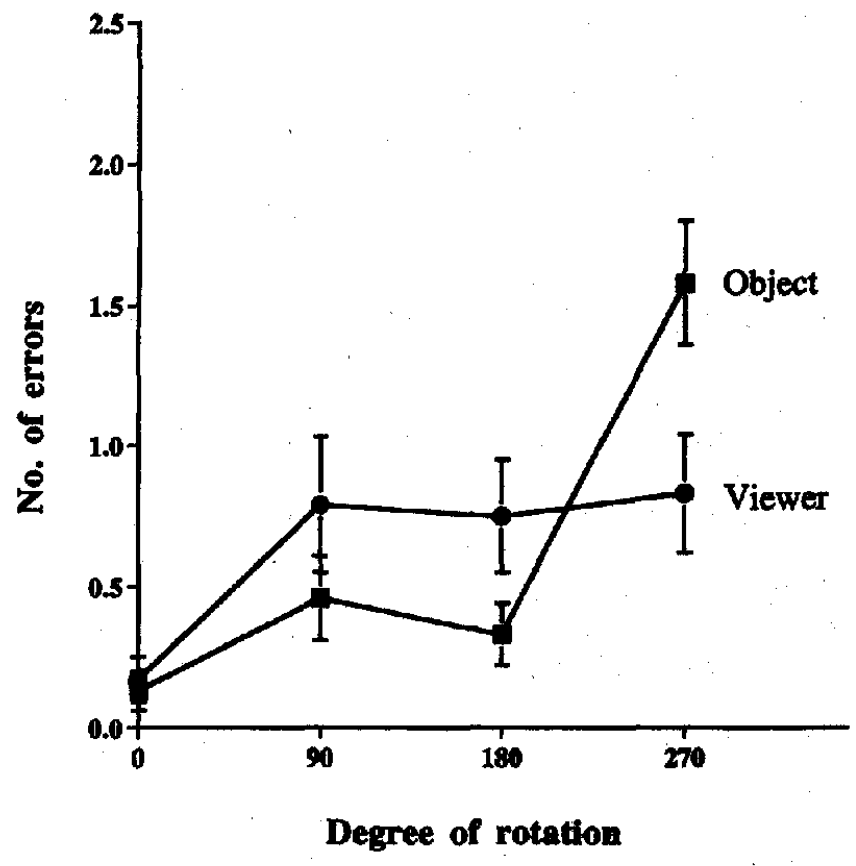

Figure 15. Mean errors and standard errors for the 16 trials, Experiment 6.

cant effect of condition. Participants responded more quickly in the block-turning condition $(M=1.90 \mathrm{~s})$ than in the control condition $(M=2.74 \mathrm{~s}), F(1,46)=4.54, p<.04$.

\section{Accuracy}

There was no difference in overall accuracy between the object ( $M=0.63$ errors) and viewer ( $M=0.64$ errors) tasks, $F(1,20)=.01, p=.93$. Figure 15 shows the mean number of errors as a function of degree. The ANOVA revealed a significant effect of degree, $F(3,60)=15.59, p<$ .0001 , a Task $\times$ Degree interaction, $F(3,60)=3.64, p<$ .02 , and a Task Order $\times$ Task interaction, $F(1,20)=6.42$, $p<.02$.

In assessing egocentric errors across tasks, we first calculated the average egocentric errors of participants who made errors in each task. This yielded similar values for both array $(M=0.85)$ and viewer $(M=1.11)$ tasks, $p=.387$. Contrary to the results of Experiments 4 and 5, the proportion of egocentric errors was elevated similarly in the array $\operatorname{task}(M=0.31)$ as in the viewer task $(M=0.42), p=.307$.

\section{Position Analysis}

As in previous experiments, right/left responses $(M=1.65 \mathrm{~s})$ were significantly slower than top/bottom responses $(M=1.50 \mathrm{~s})$ for both object and viewer tasks, $F(1,20)=10.25, p<.004$. For errors, the ANOVA revealed no overall effect of position but did reveal a significant

\footnotetext{
${ }^{10}$ The factor of rotation direction was not entered into any analyses of Experiment 6 because the block was turned only in one direction.
} 
Task $\times$ Position interaction, $F(1,20)=6.43, p<.02$. Participants made more right/left $(M=0.85$ error) than top/bottom ( $M=0.40$ error) errors in the object task, $F(1$, 23) $=9.69, p<.005$, but not in the viewer task, $p=.36$.

\section{Discussion}

In general, haptic information from a rotating block improved performance of imagined object rotations to the level of imagined self-rotations. No difference in overall accuracy was found across object and viewer tasks. Furthermore, RTs in the object task were more or less equivalent to the viewer task and were also significantly faster than those in the object control condition. In addition, the percentage of egocentric errors was elevated similarly in both tasks, contrary to the previous two object-rotation experiments.

We propose that the block-turning manipulation facilitated object performance by enabling participants to transform the representation of the object-relative frame in a cohesive manner. There are at least two possible ways this could have been achieved, both of which are compatible with the hypothesis. The first is that the block-turning manipulation may have strengthened the imagery of the on-line rotation of the object. The haptic information specified the object's orientation with respect to the observer or the environment at every phase of the transformation, which in turn may have enhanced participants' imagery of a cohesive rotation event. The second possibility is that the physical block-tuming manipulation provided haptic information for specific start- and endpoints of the block's rotation event. Facilitation may have occurred through discreet comparisons of the haptically felt end states rather than the continuous, on-line transformation of the object. This issue warrants further empirical study.

\section{General Discussion}

The present studies explored the conditions under which updating after imagined viewer and object rotations are advantageous. Participants were presented with objects or arrays of objects at one perspective and were asked to anticipate their outcome at different perspectives. Within our range of testing conditions, we found a consistent advantage for updating after imagined viewer versus object rotations.

In the first two experiments, a viewer advantage prevailed when the task involved item questions (after Presson, 1982). Participants named objects in given positions in the array after making viewer or array rotations. The viewer advantage held regardless of whether the participant was positioned outside (Experiment 1) or inside (Experiment 2) the array. In Experiment 3, the question format was changed to that of Presson's (1982) position question, where participants named the new position of a specific object. Similar to Presson's findings, performance with position questions lead to more or less equivalent performance across array and viewer tasks. However, additional catch trials, designed to test whether participants had rotated the entire array during position questions, showed that the improved array performance was most likely due to participants' ability to transform single objects rather than the entire array. The implication of this finding was that the array positionquestion task did not require imagined rotations at all: Transformations of single objects may be performed as translations. Thus, as an assessment of spatial updating during imagined rotation, the improved array result was spurious. The findings of Experiment 4 demonstrated that incorporating the four components of the array into a single encodeable object did not facilitate object rotations. However, using an object with a familiar configuration (a car) in Experiment 5 did improve object-rotation performance, albeit not to the level of viewer. The latter was more or less achieved in Experiment 6, when imagined rotations of a block were accompanied by haptic information from corresponding physical rotations.

At first glance, the persistent viewer advantage we found appears to be somewhat counterintuitive. For example, even under circumstances where participants anticipated what a small object looked like from a different view (Experiments 4 and 5), it was more efficient for them to imagine rotating themselves to the new viewpoint than to imagine rotating the object itself. However, this type of predicted-outcome task, in which no information other than the object's initial orientation is provided, has proved to be problematic for imagined object rotations. For example, Parsons (1995) found poor performance in a task where observers predicted the rotational outcome of a single Shepard-Metzler-like object. Similar results have also been reported for predicted outcomes of a rotated square (Pani, 1993, 1997; Pani \& Dupree, 1994).

Such poor performance in predicting the outcome of rotated objects is consistent with the notion that the human cognitive system has difficulties with representing cohesive transformations of the object-relative reference frame. Predicted-outcome tasks require construction of a new representation from an existing one; the new (i.e., post-rotation) representation potentially can have infinite configurations. This possibility may explain why objects appear to be encoded initially with respect to the observer's egocentric frame (Bülthoff \& Edelman, 1992; Tarr, 1995; Tarr \& Pinker, 1989; cf. Biederman \& Gerhardstein, 1993). Viewspecific encoding may facilitate spatial updating of objects by providing the cognitive system with a definitive referent against which to compare the subsequently transformed object-relative frame.

In contrast, such ambiguities appear to be absent in imagined rotations of the observer's egocentric frame, perhaps because the corresponding axes belong to the observer. It is biologically impossible to move the egocentric frame in a piecemeal manner. An argument for superior updating during imagined viewer rotations can also be grounded in considerations of everyday experience. People generally spend more time viewing objects from different viewpoints than turning objects relative to themselves. This point is reinforced by a consideration of the evolution of the human species. The fact that we have evolved as moving organisms in a mostly rigid environment-where objects rarely rotate-suggests a natural ability for updating the environment from our own viewpoint (for similar argu- 
ments, see Huttenlocher \& Presson, 1979; Simons \& Wang, 1998; Wraga, Creem, \& Proffitt, 1999a).

Our account is similar to that of Huttenlocher, Presson, and colleagues, apart from one critical distinction (Huttenlocher \& Presson, 1979; Newcombe \& Huttenlocher, 1992; Presson, 1980, 1982, 1987). They propose that objects of an array are encoded individually, with respect to the environmental reference frame. However, this proposal seems untenable for several reasons. As mentioned above, there is mounting empirical evidence that objects are initially encoded with respect to the observer rather than to the environment (Diwadkar \& McNamara, 1997; Easton \& Sholl, 1995; Franklin \& Tversky, 1990; Tar, 1995; Tarr \& Pinker, 1989). Indeed, the position analyses of the present studies generally supported Franklin and Tversky's spatial framework model of space conceptualization, which reflects the coordinate system of the physical body. Moreover, recent studies indicate that components of the environment can be selectively ignored in imagined updating (Wraga, 1998; Creem, Wraga, \& Proffitt, 1999) and that the complete absence of environmental information does not affect updating performance (Simons \& Wang, 1998).

Inconsistent with Huttenlocher, Presson, and colleagues' findings, we also found that participants' tendency to commit egocentric errors in the viewer task varied by factors other than the question type (i.e., item vs. position). Egocentric responses are those based on the primary (i.e., physical) relationship between the observer and the array, rather than the secondary (i.e., projected) relationship. We found a greater proportion of egocentric errors in viewer versus array tasks only when the array was collapsed into one object (Experiments 4 and 5), perhaps because the oneobject array afforded less turning space, which might have lead to greater overlap between physical and projected frames. Interestingly, when the object was placed on the participant's hand (Experiment 6), thus affording turning with respect to the egocentric coordinate system of the hand, the tendency to make egocentric errors was high in both tasks. These results suggest that egocentric errors are mitigated by the affordances of the array space, rather than by conflicts between primary and secondary encoding with respect to the environment. Finally, the results of Experiment 6 showed that object-rotation performance was helped and not hindered when the relationship between rotation object and environment was physically altered. Taken together, these findings evoke a simpler explanation of the processes underlying spatial updating. Problems with imagined self-rotations are associated with the behavioral potential of the space to be traversed. Problems with imagined object rotations appear to be caused by difficulties within the cognitive system in executing cohesive transformations of the object-relative reference frame.

The array/object difficulties of the present experiments were underscored in the rotation functions found consistently in those tasks. The classic rotation function established by Shepard and colleagues (e.g., Shepard \& Metzler, 1971) shows increasing latencies up to $180^{\circ}$ of rotation, with a dropoff beyond $180^{\circ}$. This result is analogous to the way objects are physically rotated, where $180^{\circ}$ is the maximum angular disparity reached before it becomes efficient to switch direction of rotation. In the present studies, array/ object latencies increased beyond $180^{\circ}$ rotations. A similar trend was found by Presson (1982), although analyses of latency differences by degree of rotation were not reported. One explanation for the results is that participants in the array/object task complied fully with the rotation instructions and imagined moving the object the full $270^{\circ}$ in one direction, even though the same participants did not complete full rotations in the viewer tasks (discussed below). Another possibility is that the shortcut strategy was implemented but overridden by the cognitive demands of the $270^{\circ}$ rotation condition. The array result is an unanticipated finding that warrants further empirical investigation.

Imagined viewer-rotation latencies also revealed unique patterns. In four of the six experiments, RTs of 90,180 , and 270 degrees were indistinguishable from one another. Previous studies of imagined viewer rotations have shown latencies to increase linearly as a function of degree of rotation (Easton \& Sholl, 1995; Rieser, 1989). By some accounts, this type of function is in fact mandatory for egocentric encoding/retrieval (Easton \& Sholl, 1995, p. 487). However, our results do not conform to this function. Nor can they be explained by reliance on other reference frames. For example, it might be argued that participants solved the viewer task by adopting a bird's eye view of the array and considering the object-relative distances between points. However, this strategy would result in no effect of degree over all rotations, including $0^{\circ}$. A more likely possibility is that participants implemented some type of egocentric strategy. For example, they may have imagined rotating the equal number of degrees to $90^{\circ}$ and $270^{\circ}$ (i.e., $90^{\circ}$ in the opposite direction) and then used a short-cut symmetry reversal strategy for $180^{\circ}$ (i.e., right $=$ left; top $=$ bottom). They also may have performed blink transformations (Kosslyn, 1994), which instantaneously transported participants to each position in the array irrespective of its distance.

The obtained differences between imagined viewer and array/object rotation functions suggests that each respective transformation may be subserved by different neural processes. This is consistent with findings from recent neuroimaging studies (Cohen et al., 1996; Kosslyn, DiGirolamo, Thompson, \& Alpert, 1998). For example, Kosslyn et al. (1998) used positron emission tomography technology to demonstrate that imagined rotations of hands activated primary motor areas of the brain corresponding to the execution of hand movements, whereas imagined rotations of Shepard-Metzler figures did not. Current investigations using functional magnetic resonance imaging will discem whether similar differences in activation are associated with imagined object and viewer rotations about an array (e.g., Creem et al., 1999).

In summary, we found a consistent advantage for spatial updating after imagined viewer versus array/object rotations. We propose that this dissociation may be due to differences in the way the corresponding reference frames of each type of rotation are transformed by the human cognitive system. Transformations of the object-relative frame 
may be affected by a general deficit within the cognitive system in representing that coordinate system as a cohesive unit. The absence of this deficit in imagined viewer transformations suggests that representations of the egocentric frame preserve the biological unity of the body.

\section{References}

Amorim, M., \& Stucchi, N. (1997). Viewer- and object-centered mental explorations of an imagined environment are not equivalent. Cognitive Brain Research, 5, 229-239.

Biederman, I., \& Gerhardstein, P. C. (1993). Recognizing depthrotated objects: Evidence for three-dimensional viewpoint invariance. Journal of Experimental Psychology: Human Perception and Performance, 19, 1162-1182.

Bryant, D. J., Tversky, B., \& Franklin, N. (1992). Internal and external spatial frameworks for representing described scenes. Journal of Memory and Language, 31, 74-98.

Bülthoff, H. H., \& Edelman, S. (1992). Psychophysical support for a two-dimensional view interpolation theory of object recognition. Pnoceedings of the National Academy of Sciences, 89, 60-64.

Cohen, M. S., Kosslyn, S. M., Breiter, H. C., DiGirolamo, G. J., Thompson, W. L., Anderson, A. K., Bookheimer, S. Y., Rosen, B. R., \& Belliveau, J. W. (1996). Changes in cortical activity during mental rotation: A mapping study using functional MRI. Brain, 119, 89-100.

Cooper, L. A. (1975). Mental rotation of random two-dimensional shapes. Cognitive Psychology, 7, $20-43$.

Creem, S. H., Hirsch, T. B., Wraga, M., Harrington, G. S., Fox, K. V., Proffitt, D. R., \& Downs III, J. H. (1999, April). FMRI investigations of inagined viewer rotations. Poster presented at the Cognitive Neuroscience Society Meeting, Washington, D.C.

Creem, S. H., Wraga, M., \& Proffitt, D. R. (1999, July). An atvantage for self- vs. object-rotations given physically impossible positions. Poster presented at the 3rd Annual Meeting of the Association for the Scientific Study of Consciousness, London, Ontario, Canada.

Diwadkar, V. A., McNamara, T. P. (1997). Viewpoint dependence in scene recognition. Psychological Science, 8, 302-307.

Easton, R. D., \& Sholl, M. J. (1995). Object-array structure, frames of reference, and retrieval of spatial knowledge. Journal of Experimental Psychology: Learning, Memory, and Cognition, 21, 483-500.

Farrell, M. J., \& Robertson, I. H. (1998). Mental rotation and the automatic updating of body-centered spatial relationships. Journal of Experimental Psychology: Learning, Memory, and Cognition, 24, 227-233.

Franklin, N., \& Tversky, B. (1990). Searching imagined environmonts. Joumal of Experimental Psychology: General, 119, 63-76.

Hardwick, D., McIntire, C., \& Pick, H. (1976). The content and manipulation of cognitive maps in children and adults. Monographs of the Society for Research in Child Development, 41(3, Serial No. 166).

Huttenlocher, J., \& Presson, C. C. (1979). The coding and transformation of spatial information. Cognitive Psychology, II, 375-394.

Howard, 1. (1982). Hraman visual orientation. Chichester, England: Wiley.

Kosslyn, S. M. (1994). Image and brain. Cambridge, MA: MIT Press.

Kosslyn, S. M., DiGirolamo, G. J., Thompson, W. L., \& Alpert, N. M. (1998). Mental rotation of objects versus hands: Neural mechanisms revealed by positron emission tomography. Psychophysiology, 35, 151-161.

Linn, M. C., \& Petersen, A. C. (1985). Emergence and characteriza- tion of sex differences in spatial ability: A meta-analysis. Child Development, 56, 1479-1498.

Macoby, E. E., \& Jacklin, C. N. (1974). The psychology of sex differences. Stanford, CA: Stanford University Press.

Newcombe, N., \& Huttenlocher, J. (1992). Children's early ability to solve perspective-taking problems. Developmental Psychology, 28, 635-643.

Pani, J. R. (1993). Limits on the comprehension of rotational motion: Mental imagery of rotations with oblique components. Perception, 22, 785-808.

Pani, J. R. (1997). Descriptions of orientation in physical ressoning. Current Directions in Psychological Science, 6, 121-126.

Pani, J. R., \& Dupree, D. (1994). Spatial reference systems in the comprehension of rotational motion. Perception, 23, 929-946.

Parsons, L. M. (1995). Inability to reason about an object's orientation using an axis and angle of rotation. Journal of Experimental Psychology: Human Perception and Performance, 21, 1259-1277.

Presson, C. C. (1980). Spatial egocentrism and the effect of an alternate frame of reference. Joumal of Experimental Child Psychology, 29, 391-402.

Presson, C. C. (1982). Strategies in spatial reasoming. Joumal of Experimental Psychology: Learning, Memory, and Cognition, 8, 243-251.

Presson, C. C. (1987). The development of spatial cognition: Secondary uses of spatial information. In N. Eisenberg (Ed.), Contemporary topics in developmental psychology (pp. 77-112). New York: Wiley.

Presson, C. C., \& Montello, D. R. (1994). Updating after rotation and translational body movements: Coordinate structure of perspective space. Perception; 23, 1447-1455.

Rieser, J. J. (1989). Access to knowledge of spatial structure at novel points of observation. Journal of Experimental Psychology: Learning, Memory, and Cognition, 15, 1157-1165.

Rieser, J. J., Guth, D. A., \& Hill, E. W. (1986). Sensitivity to perspective structure while walking without vision. Perception, 15, 173-188.

Rock, I., Wheeler, D., \& Tudor, L. (1989). Can we imagine how objects look from other viewpoints? Cognitive Psychology, 21, 185-210.

Shepard, R. N., \& Metzler, J. (1971). Mental rotation of threedimensional objects. Science, 171, 701-703.

Simons, D. J., \& Wang, R. W. (1998). Perceiving real-world viewpoint changes. Psychological Science, 9, 315-320.

Tarr, M. J. (1995). Rotating objects to recognize them: A case study on the role of viewpoint dependency in the recognition of threedimensional objects. Psychonomic Bulletin \& Review, 2, 55-82.

Tarr, M. J., \& Pinker, S. (1989). Mental rotation and orientationdependence in shape recognition. Cognitive Psychology, 21, 233282.

Wang, R. W., \& Simons, D. J. (1999). Active and passive scene recognition across views. Cognition, 70, 191-210.

Wraga, M. (1998, November). Imagined viewer rotations may defy physical laws. Poster presented at the 39th Annual Meeting of the Psychonomic Society, Dallas, TX.

Wraga, M., Creem, S. H., \& Proffitt, D. R. (1999a). The influence of spatial reference frames on imagined object- and viewer rotations. Acta Psychologica, 102, 247-264.

Wraga, M., Creem, S. H., \& Proffitt, D. R. (1999b). Spatial updating of virtual displays: Effects of extra-retinal information. Manuscript submitted for publication.

Received April 16, 1998

Revision received July 15, 1999

Accepted July 30, 1999 . 